\title{
Effect of Nitrogen and Phosphate on the Levels of Intermediates in Bakers' Yeast Grown in Continuous Culture
}

\author{
By C. M. M. FRANCO, J. E. SMITH AND D. R. BERRY* \\ Department of Bioscience \& Biotechnology, University of Strathclyde, 204 George Street, \\ Glasgow' GI IXW, UK
}

(Received 22 February 1984: revised 27 April 1984)

\begin{abstract}
Bakers' yeast (Saccharomyces cerevisiae) has been grown in continuous culture using a control medium and media which contained low levels of ammonium and phosphate. The effects of medium composition and growth rate on the levels of intermediates of the glycolytic pathways, the tricarboxylic acid cycle and the glyoxylate cycle were investigated. The energy charge varied only between 0.7 and 0.9 over the range of dilution rates studied; however, the level of ATP decreased by $50 \%$ at higher aerobic growth rates. Intermediates of the Embden-MeyerhofParnas pathway were higher at the low aerobic growth rates and decreased as the dilution rate was increased. However, higher levels of these intermediates were also observed at even higher dilution rates at which ethanol formation and fermentative metabolism occurred. Significant differences in levels of intermediates were observed between control experiments and fermentations using the low nitrogen and phosphate media. The greatest differences were observed in the levels of glucose 6-phosphate, 6-phosphogluconate, pyruvate, citrate and glyoxylate. Twenty-one different steady states were investigated and each was found to have a unique composition.
\end{abstract}

\section{INTRODUCTION}

The growth of yeast in aerobic and anaerobic conditions is of considerable commercial interest and has been extensively studied (Sols et al., 1971). Recent work has emphasized the effect of growth rate and carbohydrate status on respiratory adaptation in yeast (Barford \& Hall, 1979; Wales et al., 1980: Fiechter et al., 1981).

The level of nitrogen and phosphate in the media can markedly influence the physiological properties of bakers' yeast, particularly its activity and stability. However, it has not been possible to correlate this with any biochemically defined cell parameters (Burrows, 1970, 1979). When fermentations which produce a defined product, e.g. citric acid or penicillin, are studied, it is possible to monitor the 'fitness' of the organism by the rate of product formation. It is less easily monitored with a product such as bakers' yeast where many properties contribute to the quality of the product. However, it should be possible to correlate the 'fitness' with a given metabolic state which can be defined by the relative activity of different metabolic pathways. This can be evaluated by investigating either the level of enzymes involved in these pathways or the level of the relevant intermediates.

In these studies, we have attempted to identify the different metabolic states which may be important in determining the properties of aerobically grown yeast by measuring the level of a range of intermediates. The effect of varying growth rate and the level of nitrogen and phosphate in the medium on these metabolic states has been investigated. The levels of intermediates, however, do not give any information on flux rates through individual metabolic pathways in the cell. 


\section{METHODS}

Organism and culture media. Saccharomyces cererisiae strain GB 2333 was provided by Distillers Company (Yeast) Lid. Glenochil Technical Centre. Menstrie, UK. It was maintained on a medium containing malt extract $0.3^{\circ}$, yeast extract $0.3 \%$, glucose $1 \%$ and peptone $0.5 \%$ and stored at $4{ }^{\circ} \mathrm{C}$. Inoculum was prepared by growing the yeast in the experimental medium in a $500 \mathrm{ml}$ shaken flask and inoculating the fermenter at $1 \times 10^{6} \mathrm{cell}^{\mathrm{s}} \mathrm{ml}^{-1}$.

The experiments were carried out using a defined mineral salts medium (Oura, 1974) in which the concentration of biotin was increased to $3 \mathrm{mg} \mathrm{I} \quad$ '. Glucose, ammonium sulphate and phosphate levels were varied to give the required experimental conditions. Glucose was varied from $3 \%(w / v)$ at low dilution rate to $1 \%$ at higher dilution rates. The ratio of glucose to ammonium sulphate to phosphate in the control medium was $36: 9: 1$ and this was altered to $38: 5: 1$ in the low nitrogen medium and $125: 30: 1$ in the low phosphate medium.

Fermentation techniques. The yeast was grown in continuous culture using a Biotec FL 301 fermenter with a working volume of 3 litres. The temperature was maintained at $30 \pm 0.3{ }^{\circ} \mathrm{C}$ and the $\mathrm{pH}$ was controlled at $4.6 \pm$ 0.05 by the addition of $1.5 \mathrm{M}-\mathrm{KOH}$. Aeration was controlled up to 4 fermenter volumes of air min $^{-1}$ and oxygen tension was recorded using a Biotec glass $\mathrm{O}_{2}$ electrode. Dry weight of cells was determined by filtration through a Millipore membrane filter, pore size $2 \mu \mathrm{m}$, drying at $105^{\circ} \mathrm{C}$ for $24 \mathrm{~h}$ and cooling in a desiccator, and weighing to constant weight. Glucose in the medium was assayed by the glucose oxidase method (Bergmeyer \& Bernt, 1974) and ethanol by the alcohol dehydrogenase method of Bernt \& Gutman (1974).

Sampling technique and analysis of cellular intermediates. A $30 \mathrm{ml}$ syringe loaded with precooled (-20 $\left.{ }^{\circ} \mathrm{C}\right) 36^{\circ}$ 。 (w/v) $\mathrm{HClO}_{4}$ was used to sample the fermenter. The volume of culture removed was selected to give $0.25 \mathrm{~g}$ cell dry weight in a final concentration of $6 \% \mathrm{HClO}_{4}$. The total time for collection of the sample was <0.5 s. This method ensures rapid mixing of acid with the culture sample as turbulence is maintained throughout the sampling period. The acidified cell suspension was filtered rapidly through a Millipore Polyvic filter (pore size $2 \mu \mathrm{m}$ ) to remove the culture medium, and the cells were washed twice on the filter with $\mathrm{HClO}_{4}(6 \%)$. The leakage of intracellular metabolites during the filtration step was determined and found not to exceed $2 \%$ after $180 \mathrm{~s}$ (Franco, 1980). The concentrated washed cells were transferred into $12 \mathrm{ml} 6 \% \mathrm{HClO}_{4}$ and broken in a Braun Homogenizer (Braun. Melsungen, FRG) using glass beads (mesh size 40). Homogenization was carried out at $4000 \mathrm{r} . \mathrm{p} . \mathrm{m}$. for $45 \mathrm{~s}$ under a spray of liquid carbon dioxide to maintain the temperature between 0 and $4{ }^{\circ} \mathrm{C}$. The homogenate was centrifuged at 3500 r.p.m. for $10 \mathrm{~min}$ at $4{ }^{\circ} \mathrm{C}$ in a refrigerated centrifuge and the supernatant adjusted carefully with $5 \mathrm{M}$ $\mathrm{K}_{2} \mathrm{CO}_{3}$ to $\mathrm{pH} 5$. Samples were stored on ice and assayed immediately for metabolites. Measured amounts of metabolites were added to the acidified cell suspension prior to the homogenization step in order to evaluate the extraction procedure.

Assay of intermediates. All assays were routinely standardized using freshly prepared standard solutions of the metabolites. ATP was measured by the luciferin-luciferase method of Lundin $\&$ Thore (1975). The intensity of light produced on injection and rapid mixing of firefly-lantern-extract (FLE-250, Sigma) to the sample was measured with a Quantum photometer 951 I (Ortec, Bracknell, UK) using a logarithmic scale, and the peak height was recorded on a chart recorder. A calibration curve was produced using ATP standard solutions within the range $10^{-8}$ to $10^{-6} \mathrm{M}$-ATP. Other metabolites in the neutralized extracts were determined enzymically by coupling reactions resulting in the oxidation or reduction of NAD as described by Estabrook \& Maitra (1964). All results were expressed in $\mu \mathrm{mol}$ ( $\mathrm{g}$ dry $w \mathrm{t})^{-1}$ and are the mean of three samples. The percentage recovery for each metabolite was measured by adding known quantities to the acidified cell suspension. Values of between $83 \cdot 1 \pm$ $5.3 \%$ for ATP and $103.5 \pm 6.3 \%$ for 6 -phosphogluconate were obtained.

Chemicals. All enzymes, intermediates and co-enzymes used in these assays were obtained from Sigma: other reagents were of analytical grade from $\mathrm{BDH}$.

\section{RESULTS AND DISCUSSION}

\section{Characterization of fermentation}

Continuous cultures were successfully established using control, low nitrogen and low phosphate media. Details of the control fermentation have been published elsewhere (Franco, 1980; Berry et al., 1981) and resemble those published by other workers (Fiechter et al., 1981). In the low nitrogen and phosphate treatments, the yield of yeast was between 0.4 and $0.45 \mathrm{~g} \mathrm{(g}$ glucose $)^{-1}$ at dilution rates below $0 \cdot 2 \mathbf{h}^{-1}$. However, at higher dilution rates, the level of ethanol in the medium increased and the cell yield indicated a transition to fermentative metabolism (Fig. 1). The absence of glucose in the medium at lower dilution rates indicates that glucose was limiting at these dilution rates even when the levels of phosphate and ammonium were lowered. The maintenance energy of the aerobic glucose-limited chemostat culture was determined at $0.011 \mathrm{~g}$ glucose ( $\mathrm{g}$ dry wt biomass) ${ }^{-1}$ using the method of Pirt (1975). The values for nitrogen- and phosphate-depleted samples were 0.005 and $0.011 \mathrm{~g}$ glucose ( $\mathrm{g}$ dry wt 


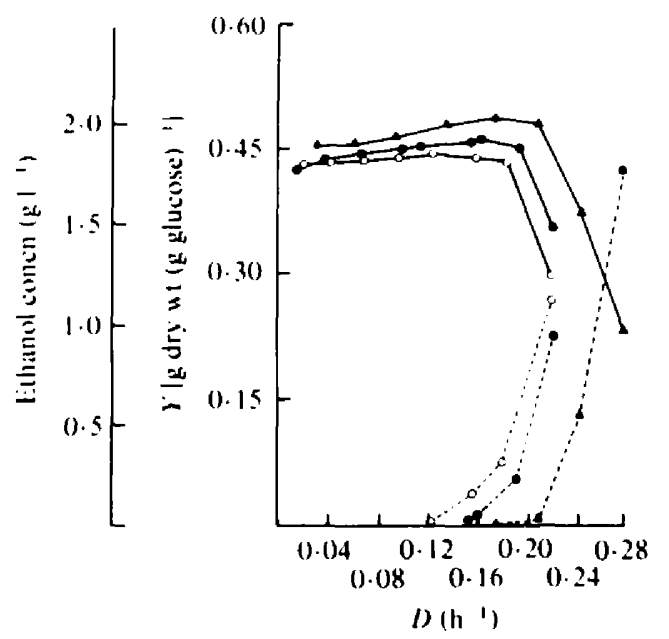

Fig. I. Yield of cells ( - ) and level of ethanol () in bakers' yeast cultures grown in continuous culture on three different media. Cells were grown on control medium ( $\boldsymbol{\Delta})$. low nitrogen medium $(\mathrm{O})$ and low phosphate medium (O).

biomass) $)^{-1}$, which correspond to dilution rates of 0.0085 and $0.009 \mathrm{~h}^{-1}$, respectively. During the fermentation with phosphate-depleted medium, the yeast acquired a pink pigmentation. Formation of a pink pigment is characteristic of disordered purine metabolism in which the yeast loses its ability to convert hypoxanthine to the riboside in the absence of biotin (Chamberlain et al., 1954: Ahmad et al., 1961). Since phosphorylation steps are essential for this pathway of purine biosynthesis, it appears that the low level of phosphate disrupts the pathway and stimulates the formation of the characteristic pigment.

\section{Levels of intermediates: adenine nucleotides and energy charge}

The concentration of intermediates was determined in triplicate at intervals once the steady state had been established. The percentage recovery of metabolites was also determined and recoveries of between 83 and $100 \%$ were obtained for all the intermediates studied (Franco, 1980). The values obtained for cellular ATP, up to $15 \mu \mathrm{mol}(\mathrm{g} \mathrm{dry} \mathrm{wt})^{-1}$ are as high as those reported previously in yeast (Chapman \& Atkinson, 1977) and indicate that the extraction and fixation technique was rapid enough to prevent significant changes in the level of intermediates during the extraction (Weibel et al., 1974).

The levels of ATP, ADP and AMP were determined and the energy charge was calculated using the method of Atkinson \& Walton (1967). In the glucose controls, the level of ATP decreased with increasing dilution rate when the yeast was grown aerobically (Fig. 2). However, it increased again at higher dilution rates, when ethanol fermentation occurred. The levels of ADP and AMP increased, reaching a maximum at the same dilution rate that gave minimum ATP values. A similar pattern was observed when low nitrogen medium was used. However, in the low phosphate medium, ADP and AMP levels were lower and did not show any increase at higher dilution rates (Fig. 2).

The energy charge varied only between 0.7 and 0.9 and the much lower values of energy charge which have been reported in yeast, especially in fermentative conditions (Ball \& Atkinson, 1975), were not observed in these experiments. Although ethanol fermentation occurred at higher dilution rates the fermentations were aerobic throughout and probably maintained some mitochondrial activity (see below). The variation of energy charge with dilution rate showed a similar pattern in the control and low nitrogen media, with a low value being observed with the onset of fermentative metabolism and the lowest ATP value. This pattern was not, however, observed in low phosphate medium in which the energy charge remained constant. 


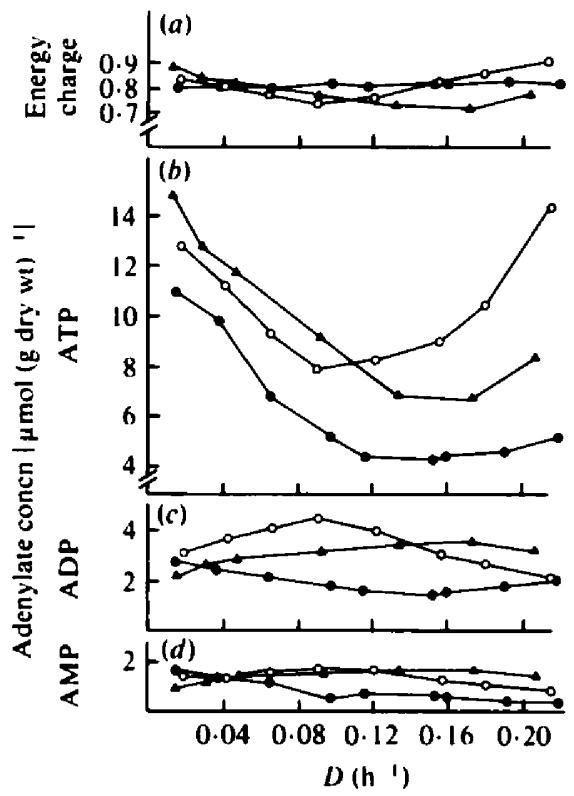

Fig. 2. Levels of (a) energy charge, (b) ATP, (c) ADP and (d) AMP obtained in cells grown in continuous culture on three difierent media, control medium $(A)$, low nitrogen medium $(O)$ and low phosphate medium (O).

The level of adenine nucleotides is subject to very complex regulatory processes (Atkinson, 1977). In Escherichia coli the energy charge remains constant over a wide range of growth rates and the higher levels of ATP are observed at the highest growth rates (for a review see Chapman \& Atkinson, 1977). However, Forrest (1965) considered that the highest levels of ATP occurred in maintenance conditions when the demand for energy for growth was at a minimum. The results we have presented in yeast appear to indicate that the lowest values of ATP and energy charge are associated with the period of highest aerobic growth rates. It should be emphasized, however, that the rate of ATP formation and breakdown is very rapid so that the level of ATP is not necessarily a good guide to its rate of production and utilization (Fiechter et al., 1981).

\section{Intermediates of glycolysis}

The metabolites of the early stages of the Embden-Meyerhof-Parnas pathway were present at high levels at low dilution rates and decreased to undetectable levels at high dilution rates. Their levels increased again, however, at dilution rates at which ethanol fermentation occurred. A similar pattern was observed with the nitrogen- and phosphate-depleted media (Fig. 3). The overall level of these intermediates was higher in cells grown in the low nitrogen medium; in particular the level of glucose 6-phosphate was high. Since both low nitrogen levels and low dilution rates have been reported to create conditions which are associated with glycogen accumulation (Kuenzi \& Fiechter, 1969, 1972), high levels of glucose 6-phosphate and fructose 6-phosphate would be anticipated in such conditions. The mass action ratio of phosphoglucose isomerase at a dilution rate of $0.0136 \mathrm{~h}^{-1}$ in the control experiments was calculated as 0.51 , which is above the equilibrium value of 0.28 and is indicative that gluconeogenesis is indeed occurring in these conditions.

In the control medium, the level of 6-phosphogluconate displayed an inverse relationship with glucose 6-phosphate, suggesting that a major part of the glucose 6-phosphate is directed to the pentose phosphate pathway. The proportion of glucose entering the pentose phosphate pathway was reported to increase with dilution rate because of the increased demand for NADPH for biosynthetic activity (Mian et al., 1974; Neijssel \& Tempest, 1976). Fructose 6-phosphate, fructose 1,6-diphosphate and the combined concentrations of dihydroxyacetone phosphate and 

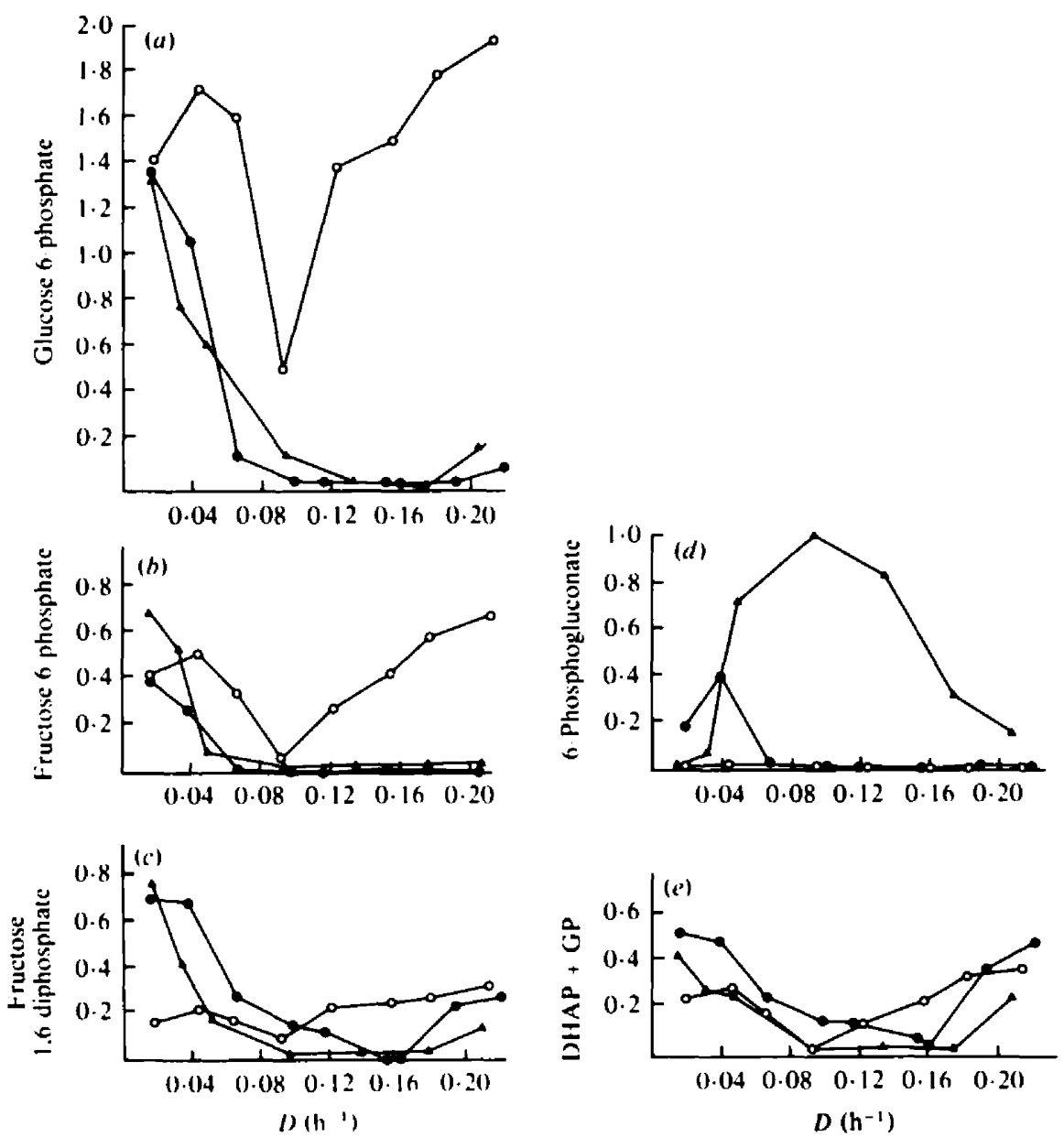

Fig. 3. Concentration of selected intermediates of the EMP and pentose phosphate pathways in yeast grown at different growth rates in continuous culture using control medium $(\boldsymbol{A})$, low nitrogen medium (O) and low phosphate medium (O). Units are $\mu \mathrm{mol}(\mathrm{g} \text { dry wt) })^{-1}$. (a) Glucose 6-phosphate, (b) fructose 6-phosphate, $(c)$ fructose 1,6-diphosphate, $(d)$ 6-phosphogluconate and $(e)$ the combined concentration of dihydroxyacetone phosphate (DHAP) and glyceraldehyde phosphate (GP).

glyceraldehyde phosphate showed a similar profile to that of glucose 6-phosphate in each of the three series of experiments.

The depletion of intermediates before triose in the Embden-Meyerhof-Parnas pathway at a dilution rate which gives the maximum rate of aerobic growth before ethanol production occurs, indicates that the onset of ethanol fermentation is not induced by the accumulation of one of these intermediates. It appears more probable that the depletion of one of these glycolytic intermediates could be important in the transition to fermentative respiration. However, this transition occurred at somewhat different dilution rates when different media were used, indicating that factors other than glucose feed rate influence this transition. In fact, by careful adaptation of the cells very high aerobic growth rates can be achieved with bakers' yeast (Barford \& Hall, 1979).

The variation in the levels of 2-phosphoglycerate, phosphoenolpyruvate and pyruvate was complex (Fig. 4). In the low nitrogen and low phosphate media the pattern resembled that of the other glycolytic intermediates, being high at low dilution rates and during fermentative growth and at a minimum during the transition phase. The level of these three intermediates in the glucose controls was somewhat lower and did not vary much at different aerobic growth rates. 

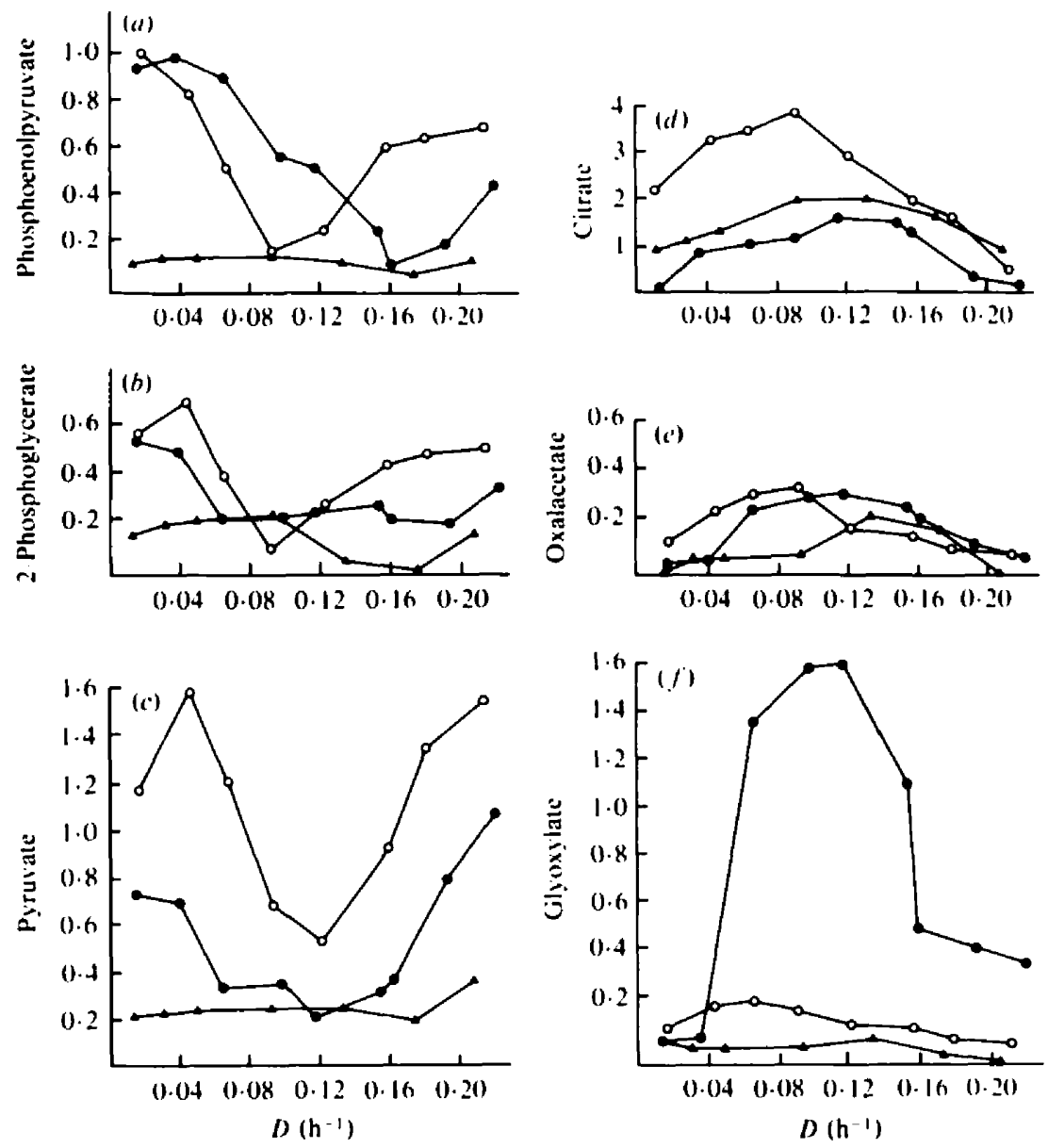

Fig. 4. Concentration of (a) phosphoenolpyruvate, (b) 2-phosphoglycerate, (c) pyruvate. (d) citrate, ( $($ ) oxalacetate and $(h)$ glyoxylate in yeast grown at different growth rates in continuous culture using control medium ( $\Delta$ ). low nitrogen medium $(O)$ and low phosphate medium (O). Units are $\mu \mathrm{mol}(\mathrm{g} \mathrm{dry}$ $(w)^{-1}$.

that is up to a dilution rate of $0.16 \mathrm{~h}$ ', but did exhibit an increase during the period of ethanol formation above this dilution rate. The high level of pyruvate and associated intermediates during ethanol fermentation could be expected since pyruvate decarboxylase in the yeast has been reported to have a higher $K_{m}$ than the pyruvate dehydrogenase enzyme (Oura, 1974).

\section{Intermediates of tricarboxylic acid and glyoxylate cycles}

The levels of citrate and oxalacetate increased with increasing dilution rate until the onset of ethanol formation, when they began to decrease. However, these intermediates persisted at a lower level in conditions which were sufficiently catabolite-repressed to form ethanol in aerobic conditions (Fig. 4). Since these results were obtained in cells grown in steady state conditions, they indicate that conditions can be established in which ethanol fermentation and tricarboxylic acid cycle functions are both present. The supply of oxygen is essential for the formation of several tricarboxylic acid cycle enzymes. Aconitase and succinate dehydrogenase for example have been reported to be produced in aerobic glucose-repressed conditions but not in anaerobic glucose-repressed conditions (Wales $e t$ al., 1980).

Depletion of phosphate and nitrogen caused only minor changes in the level of tricarboxylic acid cycle intermediates. However, whereas the level of glyoxylate remained low in the control 
and nitrogen-depleted media, it increased dramatically in the low phosphate medium. This increase may be attributable to the need for ATP and GTP for the conversion of oxalacetate to phosphoenolpyruvate during gluconeogenesis. Shortages of ATP and GTP could cause malate to accumulate and this in turn would lead to an accumulation of glyoxylate and other intermediates of the glyoxylate cycle.

One purpose of studying the physiology of industrial micro-organisms is to identify the metabolic conditions which direct most of the available nutrients into the desired product. The levels of selected intermediates of the Embden-Meyerhof-Parnas pathway, the pentose phosphate pathway, the tricarboxylic acid cycle and glyoxylate cycle are clearly influenced by both the dilution rate and the level of nitrogen and phosphate in the medium. In this study, yeast grown in twenty-one steady states which differed in either dilution rate or medium composition were investigated. In no two steady states were the levels of all the intermediates identical, so it is therefore possible to identify each one as a unique metabolic state. These metabolic states are not adequately described by the available terminology such as exponential phase or maintenance phase cells. The measurement of selected intermediates provides a valuable technique to identify and describe the growth of cells in different physiological states. We would propose from these studies that the levels of glucose 6-phosphate. 6-phosphogluconate. pyruvate, citrate and glyoxylate are valuable key indicators, whereas the levels of glyceraldehyde phosphate, dihydroxyacetone phosphate, 2-phosphoglyceric acid and oxalacetate are less useful.

The measurement of intermediates also offers a sensitive technique for studying changes in the physiological response of organisms to the environment and may be more informative than the measurement of enzyme levels in such studies (Harder \& Dijkhuizen, 1983). In the formation of bakers active dried yeast the final stages of the fermentation are very critical. The use of levels of intermediates to monitor this phase of the fermentation could give improved control.

C.M.M.F. would like to thank Distillers Company (Yeast) Lid for support throughout this work.

\section{REFERENCES}

Ahmad. F. A. Rose, A. H. \& Garg, N. K. (1961) Fffect of biotin deficiency on the synthesis of nucleic acids and proteins by $S$ caretisiat. Journal of General Microbioleg. 24, 6980.

AtKinson. D. F. (1977). Cellutar Einerg! Me'uholixm. New York: Academic Press.

AtKinson. D. F. \& Walton, (;. M. (1967). Adenosine triphosphate conservation on metabolic regulation. Journal of Biological (hemistry 242. 32393341.

Ball. W. J. \& Atkissox. D. F. (1975). Adenylate charge in Saccharomeces cere'risiae during starvation. Journal of Bacteriolog! 121. 975982.

BARFord. J. P. \& Hall, R. J (1979). An examination of the Crabtree effect in Saccharomice's cercecisises: the role of respiratory adaptation. Journal of Gieneral Ificrobiology 114, 267275

Bergimeyer. H. U \& BerNt. F. (1974). Determination with glucose oxidase and peroxidase. In Metherds of Enz:matic Analysis, vol. 3. pp. 1205 1215. Fdited by H. U. Bergmeyer. Neu York: Academic Press

BerNt. F. \& Gltman. I. (1974) Determination with alcohol dehydrogenase and NAD. In Metheds of Enzymatic Analisis, vol. 3. pp 1499 150)2. Fdited by H. U. Bergmeyer. New York: Acidemic Press

BERRY. D. R. SMITH. J. F. \& Franco. ( . M. M. (1981). Intermediate levels in bakers yeast grown in continuous culture. In Curre'm De'telopments in least Research. pp. 393 398. Fdited by G. G. Stewart \& J Russell. Toronto: Pergamon.
Bitrows. S. (1970). Bakers Yeast. In The Yeasls, vol. 3. pp. $\$ 49420$. Edited by A. H. Rose \& J. S. Harrison. L.ondon \& New York: Academic Press. Blrkows, S. (1979). Bakers Yeast. In Ecomomic Microhiolsgi: vol. 4. pp. 31 64. Edited by A. H. Rose. London \& New York: Academic Press.

(hamberlain. N.. Cutts, N. S. \& Rainbow. C. (1954). The formation of pigment and arylamine by yeasts. Journal of General Microhishog. 7. 54 60).

( hapMaN, A. G. \& AtKINSON. D. F. (1977). Adenine nucleotide concentrations and turnover rates. their correlation with biological activity in bacteria and yeast. Adrances in Microhiol Physiologl 15. 253306.

Fstabrook. R. W. \& Maltra. P. K. (1964). A fluorimetric method for the quantitative microanalysis of adenine pyridine nucleotides. Analytical Biochemistry 3, 369,382

Fiechter. A.. FlhrmaNi, G. F. \& Kappeli, O. (1981). Regulation of glucose metabolism in growing yeast cells. Adrame's in Microhial Phisiolog! 22. 12318.3.

Forrest, W. W. (1965). ATP pool during the growth cycle of Sireptococe us fatecalis. Journal of Bacteriology. 90. 10131016 .

Fravco. (. M. M. (1980). Studies en intermediary. metatulism of hakers geast during aerobic growth. PhD thesis. University of Strathclyde.

Harder. W. \& Dukhtizen, L. (1983). Physiological responses to nutrient limitation. Anmual Revie's of Microhiolog. 37. I 23. 
Kuenzi, M. T. \& Fiechter, A. (1969). Changes in carbohydrate composition and trehalose during the budding cycle of Saccharomyces cerevisiae. Archives of Microbiology 64, 396407.

Kuenzi, M. T. \& Fiechter, A. (1972). Regulation of carbohydrate composition of Saccharomyces cerevisiae under growth limitation. Archices of Microbiolog.' 84. 254-262.

LUNDIN, A. \& ThORE, A. (1975). Comparison of methods for extraction of bacterial adenine nucleotides determined by firefly assay. Applied Microbiology' 30, 713-721

Mian, F. A., Fencl, S., Prokop, A., Monagegeghi, A. \& FAzELI. A. (1974). Effect of growth rate on glucose metabolism of yeast grown in continuous culture. Folia microbiologica 19, 191-198.

Neijssel. O. M. \& Tempest, D. W. (1976). The role of carbohydrate metabolism in Klebsiella aerogenes NCTC 418 organism growing in a chemostal culture. Archires of Microbiology 106, 251-258.
OURA, E. (1974). Effect of aeration intensity on the biochemical composition of bakers yeast. I. Factors affecting the type of metabolism. Biotechnology and Bioengineering 16, 1197-1212.

PIRT. S. J. (1975). Principles of Microbe and Cell Cultiration. London: Blackwell Scientific.

Sols. A., Gancedo, C. \& Delafuente, G. (1971). Energy yielding metabolism in yeast. In The Yeasts. vol. 2, pp. 271-367. Edited by A. H. Rose \& J. S. Harrison. London \& New York: Academic Press.

Wales, D. S.. Cartledge, T. G. \& Lloyd, D. (1980). The effect of glucose repression and anaerobiosis on the activities and subcellular distribution of TCA cycles and associated enzymes in Saccharomyces carlsbergensis. Journal of General Microbiology 116, 93-98.

Weibel, K. E.. MOR, J. R. \& Fiechter, A. (1974). Rapid sampling of yeast cells and automated assays of adenylate, citrate, pyruvate and glucose 6phosphate pools. Analitical Biochemistry 58, 208 216. 